Archive for

Organic Chemistry

Arkivoc 2017, part iii, 335-345

\title{
Synthesis of novel heterocyclic fused pyrimidin-4-one derivatives from imino-1,2,3-dithiazoles
}

\author{
Maria de Fatima Pereira, ${ }^{a, b}$ Elena Sarghe, ${ }^{b}$ Hervé Rouillard, ${ }^{b}$ Lisianne Domon, ${ }^{b}$ \\ Jean-René Chérouvrier, ${ }^{b}$ and Valérie Thiéry ${ }^{b^{*}}$ \\ a Normandie Université, UNICAEN, CERMN (Centre d'Etudes et de Recherche sur le Médicament de \\ Normandie), F-14032, Caen, France \\ ${ }^{b}$ Université de La Rochelle, UMR CNRS 7266 -LIENSS, LIttoral ENvironnement SociétéS, Faculté des Sciences et \\ Technologie, Avenue Michel Crépeau, 17042 La Rochelle, France \\ E-mail: vthiery@univ-Ir.fr
}

Dedicated to Prof. Oleg Rakitin on the occasion of his 65th birthday

Received 07-09-2017

Accepted 08-27-2017

Published on line 10-11-2017

\section{Abstract}

Extending the potential applications of 4,5-dichloro-1,2,3-dithiazolium chloride chemistry, we investigated the synthesis of original derivatives of thieno[2,3-d]pyrimidin-4-one system by condensation of alkyl and aromatic diamines with 2- $\mathrm{N}$-iminodithiazolothiophene derivatives. We continued our study for access to novel pyrazoloand pyrido- fused pyrimidinones using the potential applications of Appel's salt chemistry.

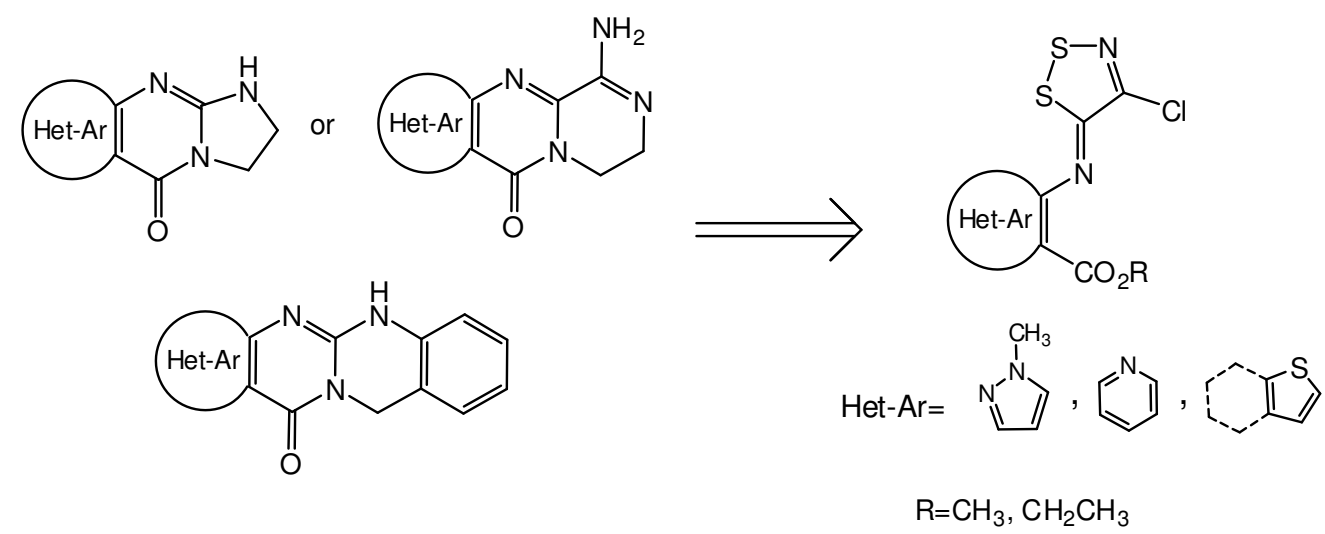

Keywords: Imino-1,2,3-dithiazoles, Appel's salt, thieno[2,3-d]pyrimidin-4-ones, heterocyclic fused pyrimidin-4ones 


\section{Introduction}

Nitrogen-containing heterocycles with a sulfur atom are an important class of compounds in medicinal chemistry. The thienopyrimidinone nucleus is known to be a pharmacologically relevant structure in medicinal chemistry and has generated interest from many research groups on account of its useful biological properties. For instance, the thieno[2,3- $d$ ]pyrimidinone substructure exhibits anticancer, ${ }^{1-5}$ antimalarial $^{6}$ and antitubercular ${ }^{7}$ activities. Some thieno[2,3- $d$ ] pyrimidin-(4)-ones act as selective phosphodiesterase inhibitors for the treatment of inflammatory diseases, ${ }^{8,9}$ antagonists of the glutamate receptors ${ }^{10}$ whereas others were identified as highly selective SIRT2 inhibitors. ${ }^{11,12}$ Owing to their growing use in compounds of therapeutic importance, the synthesis of various thienopyrimidinone-based molecules has been actively pursued in the last past decade and studied in detail, leading to several new developments. ${ }^{3,13,14}$ This skeleton is usually obtained by condensation and ring closure from various 2,3-substituted thiophenes such as Gewald's amide, ${ }^{15}$ 2-aminothiophene-3-carbonitrile, ${ }^{16} \quad$ 3-carbethoxy-2-phenylthioureathiophene, ${ }^{17} \quad$ 2-aminothiophene-3carboxylic ester. ${ }^{18}$

It is well known that reaction of 4,5-dichloro-1,2,3-dithiazolium chloride (2) (Appel's salt) with primary aromatic amines allows access to $N$-arylimino-1,2,3-dithiazoles, usually in high yield. ${ }^{19,20}$ These imines have proved to be very versatile synthetic intermediates in heterocyclic chemistry, undergoing a variety of reactions initiated by nucleophilic attack at different sites on the dithiazole ring (Scheme 1). ${ }^{21-23}$

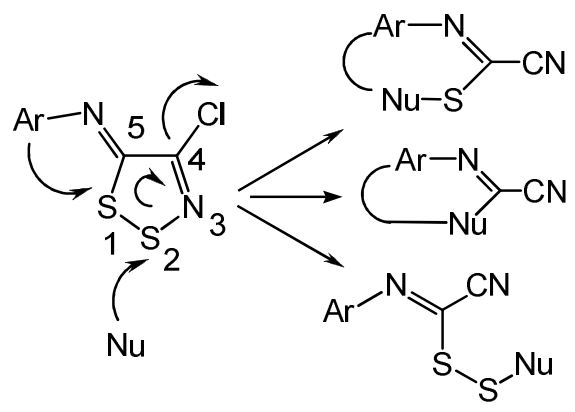

Scheme 1. Chemical transformations of $\mathrm{N}$-arylimino-1,2,3-dithiazoles.

As a part of our ongoing research studies concerning the preparation and biological evaluation of sulphur and nitrogen-containing novel heterocycles, we have already been involved in exploiting the potential synthetic applications of 5-arylimino 4-chloro-5H-1,2,3-dithiazoles ${ }^{24}$. Kim and co-workers ${ }^{25,26}$ followed by us reported the conversion of methyl 3- $\mathrm{N}$-(4-chloro-5H-1,2,3-dithiazol-5-ylidene)thiophene-2-carboxylate $\mathbf{V}$ with various alkyl and aromatic diamines into thieno[3,2-d]pyrimidin-4-one derivatives I, II, III, IV (Scheme 2.) $)^{27,28}$

However, no reports are available so far on the synthesis of thieno[2,3- $d$ ]pyrimidin-4-ones from imino1,2,3-dithiazoles. Development of new original synthetic routes to this type of molecules remains a challenging task of current interest.

For the reason given above, we have investigated the synthesis of novel thieno[2,3- $d$ ]pyrimidinone derivatives using the potential applications of Appel's salt chemistry. 
<smiles>C=C(C)Cn1ccc(N=c2ssnc2Cl)c1C(=O)O</smiles>

Scheme 2. Polycyclic thieno[3,2- $d$ ] pyrimidinones structurally close to Rutaecarpine alkaloid synthesized from methyl $\mathrm{N}$-(4-chloro-5H-1,2,3-dithiazol-5-ylidene)thiophene-3-carboxylate.

\section{Results and Discussion}

Following the usual methods, ${ }^{24}$ treatment of commercially available ethyl 2-amino-4,5,6,7tetrahydrobenzo[b]thiophene-3-carboxylate (1) with 4,5-dichloro-1,2,3-dithiazolium chloride (Appel's salt) (2) in dichloromethane at room temperature afforded $68 \%$ of the corresponding iminodithiazole (3) (Scheme 3). The presence of the fused cyclohexane ring avoids unwanted addition of Appel's salt at position C-5 of the thiophene.

Considering our previously published results ${ }^{27,28}$ and those from Kim's group, ${ }^{25,26}$ we decided to investigate the reaction of the new iminodithiazole with ethylenediamine and 0 -aminobenzylamine in order to access to original structures containing thieno[2,3- $d$ ]pyrimidin-4-one ring.

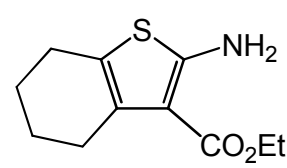

1

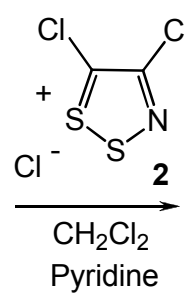

r.t.

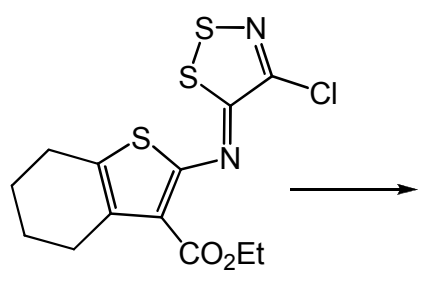

$3(68 \%)$<smiles>NC1=NCCn2c1nc1sc3c(c1c2=O)CCCC3</smiles>

$4(10 \%)$<smiles>N#CCCn1c(CCN)nc2sc3c(c2c1=O)CCCC3</smiles><smiles>COC(=O)c1c(NC(=S)C#N)sc2c1CCCC2</smiles>

$5(63 \%)$

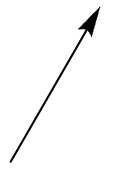

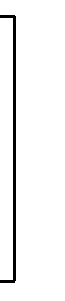

Scheme 3. Synthesis of iminodithiazole (3) and conversion into the new tetracyclic thieno[2,3- $d$ ]pyrimidinone derivative (4). 
We discovered that stirring imine with ethylene diamine in THF at low temperature allowed the rapid synthesis of the original heterocyclic skeleton (4) (10\% yield) accompanied by a large amount of the cyanothioformamide (5) (63\%). Considering the previous results published by our team, ${ }^{27,28}$ the most plausible mechanism of the reaction implied existence of a key intermediate (VI) and a final nucleophilic attack of the primary amino group of ethylene diamine on the carbonitrile carbon to generate the cyclic amidine (Scheme 3). Pursuing our strategy, the iminodithiazole precursor (3) was heated under microwaves irradiation (at $120^{\circ}$ ) with 0 -aminobenzylamine and yielded $7 \%$ of new pentacyclic skeleton (6) and a mixture of two other products which were identified as the new 2-thiocarboxamidothiophene (7) (22\%) and the known cyanothioformamide (5) (11\%).

It should be noticed that the resulting product (6) was difficult to isolate and the yield was quite low (7\%), showing the difficulty for the intermediate to cyclize. Herein, formation of the pentacylic core (6) suggested nucleophilic substitution of the cyano group of the intermediate (VII) by the o-aminobenzylamine (Scheme 4).

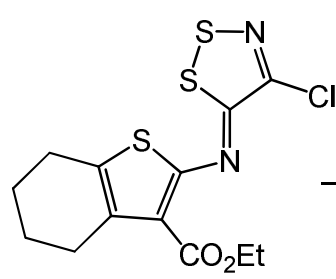

3

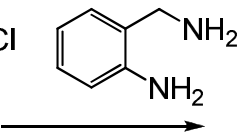<smiles>O=c1c2c3c(sc2nc2n1Cc1ccccc1N2)CCCC3</smiles>

$6(7 \%)$<smiles>COC(=O)c1c(NC(=S)c2ncc3ccccc3n2)sc2c1CCCC2</smiles>

$7(22 \%)$

$+$

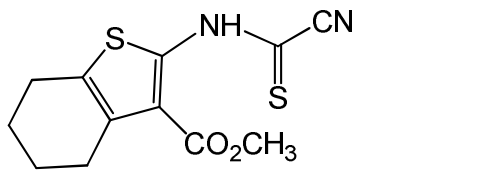

$5(11 \%)$

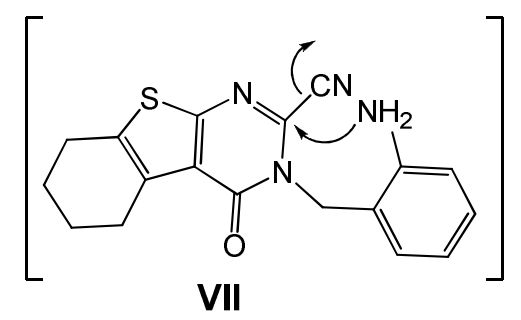

Scheme 4. Synthesis of the novel pentacyclic thieno[2,3-d]pyrimidinone system (6).

In continuation of this work, we attempted to isolate the methyl 2- $\mathrm{N}$-(4-chloro-5H-1,2,3-dithiazol-5ylidene)thiophene-3-carboxylate counterpart. To the best of our knowledge, the chemical behaviour of this iminodithiazole with amines has never been reported.

We found that standard methods at room temperature ${ }^{24}$ applied to the preparation of 2-Niminodithiazolothiophene derivatives led to a complex mixture of polymeric oily compounds resulting from electrophilic substitution at C-5 of the thiophene ring by N-1 of Appel's salt (2). Nevertheless, condensation of the starting amino ester (8) with one equivalent of Appel's salt (2) in dichloromethane at low temperature (-20 ${ }^{\circ} \mathrm{C}$ ) followed by addition of pyridine (2 equiv.) yielded $6 \%$ of rare methyl 2- $\mathrm{N}$-(4-chloro-5H-1,2,3-dithiazol-5ylidene)thiophene-3-carboxylate (9) (Scheme 5). 

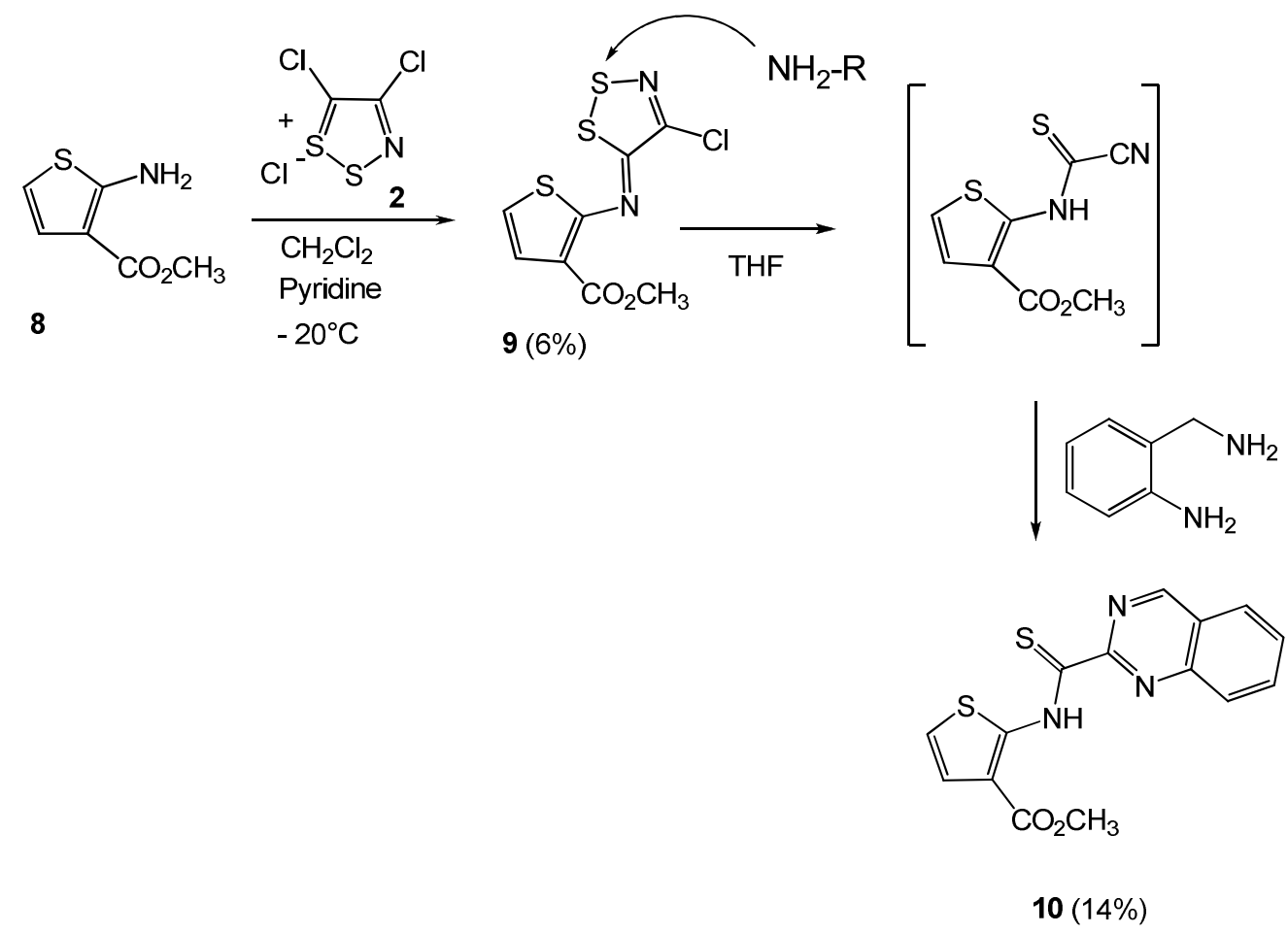

Scheme 5. Synthesis of methyl $\mathrm{N}$-(4-chloro-5H-1,2,3-dithiazol-5-ylidene)thiophene (9) and conversion into the new 2-thiocarboxamide derivative (10).

We decided to investigate the reaction of imine (9) with 0 -aminobenzylamine (2 equiv.). Under heating in THF, a rather complex mixture was formed from which only the quinazoline-2-thiocarboxamide (3-carbethoxy2-(quinazolinyl)thiocarboxamido thiophene) (10) was isolated in $14 \%$ yield. Whatever the experimental conditions, exposing the obtained imine (9) with diamine under microwave irradiation, neat or in a sealed vial, or in various solvent were unsuccessful to give the expected tetracyclic compound.

Furthermore, we have extended this methodology towards new polycyclic analogs, using ethyl 5-amino-1methyl-1H-pyrazole-4-carboxylate and methyl 2-aminopyridine-3-carboxylate as readily available heteroaromatic substrates. Corresponding imines (11) and (12) were obtained in dichloromethane at room temperature with respectively $68 \%$ and $51 \%$ yields. Under the same conditions reported to those employed above, treatment of imines with alkane and aromatic diamines led to new heterocyclic fused pyrimidinones (Scheme 6). The reaction of imines with ethylene diamine afforded the cyclized and stable products (13 and $14)^{29,30}$ which result from the substitution of the cyano group by nucleophilic attack of the aliphatic amine of the intermediate (VIII) rather than the expected cyclic amidine described above.

Finally, only the tetracyclic compound (15) was identified in $24 \%$ yield when imine (11) reacted with oaminobenzylamine. Compound (12) containing pyrimidine core did not react with aromatic diamine to give the target tetracyclic product. 


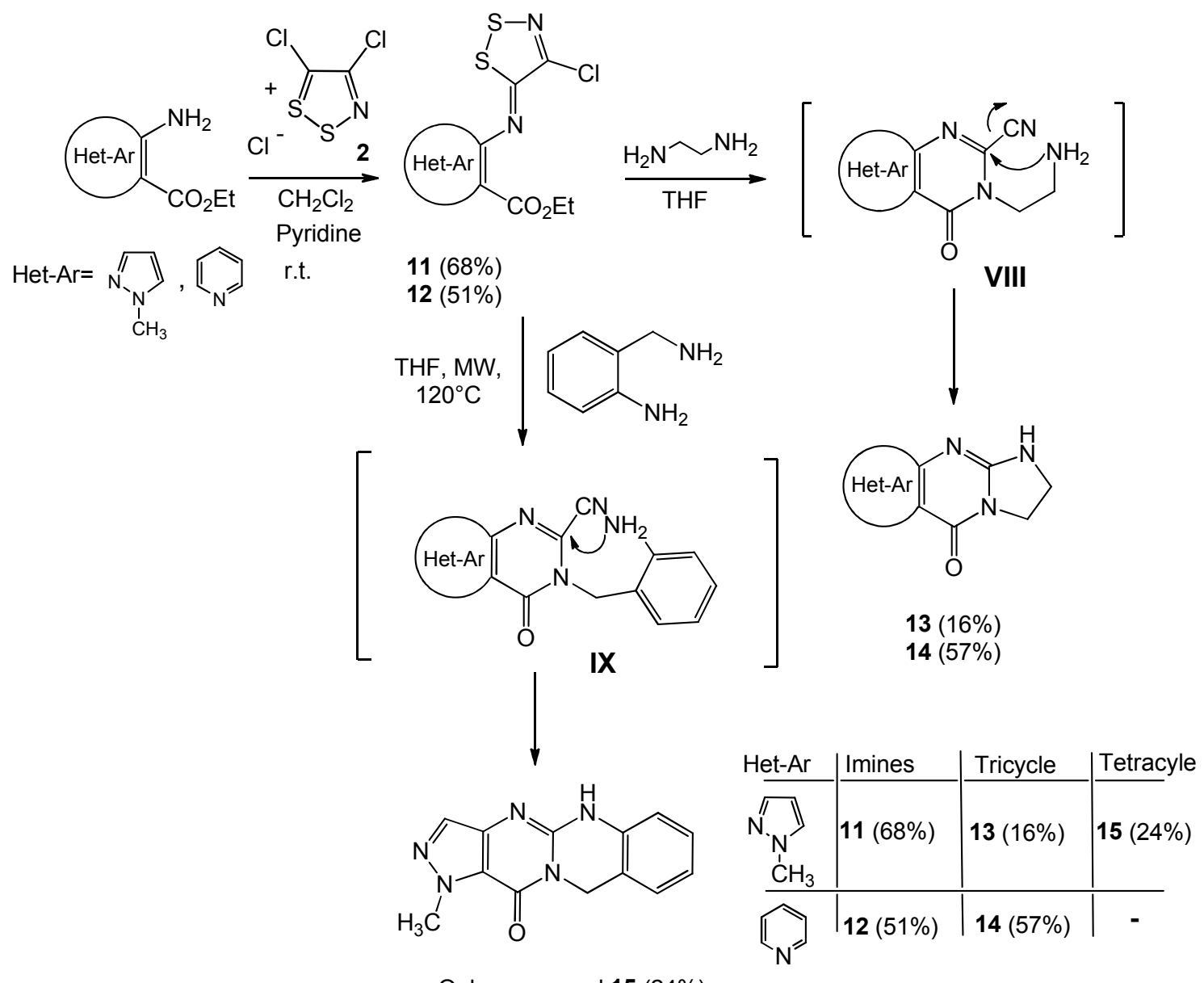

Only compound $15(24 \%)$

Scheme 6. Synthesis of new heterocyclic fused pyrimidinones.

\section{Conclusions}

We described here a method which allows access to complex fused products starting from easily obtainable substrates in one step. We demonstrated that 5-( $\mathrm{N}$-arylimino)-4-chloro-5 $\mathrm{H}$-1,2,3-dithiazole derivatives can be used as available building blocks for the rapid synthesis of various polycyclic molecules. The pharmacological targets of these original heterocycles remain to be established.

\section{Experimental Section}

General. All commercially available compounds were used as received without further purification. Silica gel 0.063-0.2 mm (70-230 mesh) was used for all column chromatography. NMR spectra were recorded on a Jeol NMR LA400 spectrometer in chloroform-d or DMSO- $d_{6}$ at $400 \mathrm{MHz}$ for ${ }^{1} \mathrm{H}$ NMR spectra and $100 \mathrm{MHz} \mathrm{MHz}$ for ${ }^{13} \mathrm{C}$ NMR spectra. Chemical shifts were reported in ppm and multiplicities were described as follows: bs, broad singlet; s, singlet; $d$, doublet; $t$, triplet; q, quartet; $m$, multiplet. Coupling constants ' $\mathrm{J}$ ' were reported in $\mathrm{Hz}$. IR spectra were recorded on a Perkin Elmer spectrum 100 FT-IR ATR spectrometer. Absorptions are given in wavenumbers $\left(\mathrm{cm}^{-1}\right)$. Melting points were determined on a Kofler melting point apparatus. Mass spectra were 
measured with a Micro mass Q-TOF spectrometer. Microwave experiments were conducted in sealed vials ( 10 $\mathrm{mL}$ ) with a Biotage Initiator microwave reactor ( $400 \mathrm{~W}$, monomode system with a microwave power delivery system ranging from 5 to 400 W.) under air with magnetic stirring. Reaction temperature and pressure were determined using the built-in, on-line IR and pressure sensors.

General procedure for the synthesis of imino-1,2,3-dithiazoles 3, 9, 11 and 12 from starting amino ester derivatives. $^{25-28}$ Under an inert atmosphere (argon), dithiazolium salt $1(0.208 \mathrm{~g}, 1 \mathrm{mmol})$ was added to a solution of amino ester $(1 \mathrm{mmol})$ in dichloromethane $(5 \mathrm{~mL})$. Pyridine $(2 \mathrm{mmol})$ was slowly added. The mixture was stirred until all of the amine had been consumed (tlc control). The mixture was warmed to room temperature and the reaction mixture filtered through acidic alumina and pour into ice water and the organic layer was separated and the aqueous phase extracted with dichloromethane. The crude product was purified by column chromatography using petroleum ether/DCM as the eluent.

2-[4-Chloro-[1,2,3]dithiazol-(5Z)-ylideneamino]-4,5,6,7-tetrahydro-benzo[b]thiophene-3-carboxylic acid ethyl ester (3). Orange solid; Yield: $68 \%$; mp $130{ }^{\circ} \mathrm{C} ;{ }^{1} \mathrm{H} \mathrm{NMR}\left(400 \mathrm{MHz}, \mathrm{CDCl}_{3}\right) \delta: 4.38\left(\mathrm{q}, 2 \mathrm{H}, J 7.2 \mathrm{~Hz}, \mathrm{CH}_{2}\right)$, 2.72-2.92 (m, 4H, $\left.2 \mathrm{CH}_{2}\right), 1.73-2.00\left(\mathrm{~m}, 4 \mathrm{H}, 2 \mathrm{CH}_{2}\right), 1.41\left(\mathrm{t}, 3 \mathrm{H}, J 7.2 \mathrm{~Hz}, \mathrm{CH}_{3}\right) .{ }^{13} \mathrm{C} \mathrm{NMR}\left(100 \mathrm{MHz}^{\mathrm{CDCl}}\right)_{3} \delta:$ 164.0; 152.0; 149.2; 148.9; 136.4; 133.1; 127.2; 60.8; 25.7; 25.6; 22.9; 22.3; 14.3. $U_{\max }(\mathrm{ATR}) / \mathrm{cm}^{-1}: 2929$, $1665,1545,1268,1146,779$. HRMS (ESI) $\mathrm{m} / \mathrm{z}[\mathrm{M}+\mathrm{H}]^{+}$: calcd for $\mathrm{C}_{13} \mathrm{H}_{13}{ }^{35} \mathrm{CIN}_{2} \mathrm{O}_{2} \mathrm{~S}_{3}$ : 359.98277 ; found : 359.9853.

2-[4-Chloro-[1,2,3]dithiazol-(5Z)-ylideneamino]-thiophene-3-carboxylic acid methyl ester (9). Yellow solid; Yield: 6\%; mp $152{ }^{\circ} \mathrm{C} ;{ }^{1} \mathrm{H} \mathrm{NMR}\left(400 \mathrm{MHz}, \mathrm{CDCl}_{3}\right) \delta: 7.56(d, 1 \mathrm{H}, J 5.4 \mathrm{~Hz}$, Harom), 7.29 (d, $1 \mathrm{H}, J 5.4 \mathrm{~Hz}, \mathrm{Harom})$, $3.92\left(\mathrm{~s}, 3 \mathrm{H}, \mathrm{CH}_{3}\right) .{ }^{13} \mathrm{C}$ NMR $\left(100 \mathrm{MHz}_{2} \mathrm{CDCl}_{3}\right) \delta: 162.7 ; 155.3 ; 153.8 ; 148.6 ; 129.3 ; 125.3 ; 119.1 ; 51.6 . U_{\max }$ (ATR) $/ \mathrm{cm}^{-1}: 3103,2952,2922,1668,1285,700$. HRMS (ESI) $\mathrm{m} / z[\mathrm{M}+\mathrm{H}]^{+}:$calcd for $\mathrm{C}_{8} \mathrm{H}_{5}{ }^{35} \mathrm{CIN}_{2} \mathrm{O}_{2} \mathrm{~S}_{3}: 291.92017$; found : 291.9211.

5-[4-Chloro-1,2,3] dithiazol-(5Z)-ylideneamino]-1-methyl-1H-pyrazole-4-carboxylic acid ethyl ester (11). Yellow solid; Yield: 68\%; mp $152{ }^{\circ} \mathrm{C} ;{ }^{1} \mathrm{H}$ NMR $\left(400 \mathrm{MHz}, \mathrm{CDCl}_{3}\right) \delta: 7.93(\mathrm{~s}, 1 \mathrm{H}, \mathrm{H}), 4.25\left(\mathrm{q}, J 7.2 \mathrm{~Hz}, 2 \mathrm{H}, \mathrm{CH}_{2}\right)$, $3.76\left(\mathrm{~s}, 3 \mathrm{H}, \mathrm{CH}_{3}\right), 1.28\left(\mathrm{t}, J 7.2 \mathrm{~Hz}, 3 \mathrm{H}, \mathrm{CH}_{3}\right) .{ }^{13} \mathrm{C} \mathrm{NMR}\left(100 \mathrm{MHz}, \mathrm{CDCl}_{3}\right) \delta: 165.0 ; 162.0 ; 149.5 ; 147.5 ; 141.4$; $102.0 ; 60.1 ; 35.5 ; 14.2$. $U_{\max }\left(\right.$ ATR) $/ \mathrm{cm}^{-1}: 2978,1701,1587,1056,771$. HRMS (ESI) $\mathrm{m} / z$ [M+Na] ${ }^{+}$calcd for $\mathrm{C}_{9} \mathrm{H}_{9}{ }^{35} \mathrm{ClN}_{4} \mathrm{O}_{2}: 326.9753$; found : 326.9750 .

2-[4-Chloro-[1,2,3]dithiazol-(5Z)-ylideneamino]-nicotinic carboxylic acid methyl ester (12). Yellow solid; Yield: 51\%; mp $125^{\circ} \mathrm{C} ;{ }^{1} \mathrm{H} \mathrm{NMR}\left(400 \mathrm{MHz}^{\mathrm{C} C \mathrm{CD}_{3}}\right) \delta: 8.71(\mathrm{dd}, J 2 \mathrm{~Hz}, J 4.8 \mathrm{~Hz}, 1 \mathrm{H}, \mathrm{H}), 8.33(\mathrm{dd}, J 2 \mathrm{~Hz}, J 7.4 \mathrm{~Hz}$, $1 \mathrm{H}, \mathrm{H}), 7.37$ (dd, J $4.8 \mathrm{~Hz}, J 7.4 \mathrm{~Hz}, 1 \mathrm{H}, \mathrm{H}), 4.03\left(\mathrm{~s}, 3 \mathrm{H}, \mathrm{CH}_{3}\right) .{ }^{13} \mathrm{C} \mathrm{NMR}\left(100 \mathrm{MHz}, \mathrm{CDCl}_{3}\right) \delta: 166.1 ; 159.2$; $152.2 ; 149.8 ; 146.3 ; 139.9 ; 124.0 ; 120.9 ; 52.7 . U_{\max }(\mathrm{ATR}) / \mathrm{cm}^{-1}: 2946,1686,1524,1411,776$. HRMS (ESI) $\mathrm{m} / \mathrm{z}[\mathrm{M}+\mathrm{H}]^{+}$: calcd for $\mathrm{C}_{9} \mathrm{H}_{6}{ }^{35} \mathrm{ClN}_{3} \mathrm{O}_{2} \mathrm{~S}_{2}: 287.9668 ;$ found : 287.9668 .

General procedure for reaction of iminodithiazoles with 1,2-ethylene diamine, synthesis of compounds 4,13 and 14. A solution of ethylene diamine $(0.04 \mathrm{~mL}, 0.55 \mathrm{mmol})$ in tetrahydrofuran (THF) (5 mL) was added slowly to a solution of imino-1,2,3-dithiazole (compounds 3, 9, 11 and 12) (0.55 mmol) in THF (15 mL). The mixture was stirred at low temperature under inert atmosphere (argon) until all of the imine had been consumed (tlc control). After evaporation of the solvent under reduced pressure, column chromatography on silica gel with dichloromethane/ethanol $(9: 1, \mathrm{v} / \mathrm{v})$ as solvents gave products $\mathbf{4 , 5 , 1 3}$ and 14 as solids.

1-Amino-2,3,4,7,8,9,10-heptahydro-6H-[1]benzothieno[2,3-d]pyrazino[1,2-a]pyrimidin-6-one (4). White solid; Yield: $10 \%$; $\mathrm{mp} 218{ }^{\circ} \mathrm{C},{ }^{1} \mathrm{H}$ NMR $\left(400 \mathrm{MHz}, \mathrm{DMSO}-d_{6}\right) \delta: 6.37\left(\mathrm{~s}, 2 \mathrm{H}, \mathrm{NH}_{2}\right), 3.96\left(\mathrm{t}, J 6,8 \mathrm{~Hz}, 2 \mathrm{H}, \mathrm{CH}_{2}\right), 3.57$ $\left(\mathrm{t}, J 6.8 \mathrm{~Hz}, 2 \mathrm{H}, \mathrm{CH}_{2}\right), 2.92\left(\mathrm{~d}, J 5.7 \mathrm{~Hz}, 2 \mathrm{H}, \mathrm{CH}_{2}\right), 2.78\left(\mathrm{~d}, J 5.7 \mathrm{~Hz}, 2 \mathrm{H}, \mathrm{CH}_{2}\right), 1.86-1.74\left(\mathrm{~m}, 4 \mathrm{H}, 2 \times \mathrm{CH}_{2}\right) .{ }^{13} \mathrm{C} \mathrm{NMR}$ $\left(100 \mathrm{MHz}, \mathrm{DMSO}-d_{6}\right) \delta: 159.5 ; 156.2 ; 151.2 ; 139.9 ; 134.9 ; 131.2 ; 122.1 ; 42.7 ; 37.9 ; 25.2 ; 24.7 ; 22.3$; 
21.7. $U_{\max }(A T R) / \mathrm{cm}^{-1}: 3464,2922,1655,1537,1348,1153,779,521$. HRMS (ESI) $\mathrm{m} / z$ [M+H] $]^{+}$calcd for C13H14N4OS : 275.0966 ; found : 275.0968 .

2-(Cyanocarbothioyl-amino)-4,5,6,7-tetrahydro-benzo[b]thiophene-3-carboxylic acid ethyl ester (5). Orange solid; Yield: 63\%; mp $140{ }^{\circ} \mathrm{C} ;{ }^{1} \mathrm{H}\left(\mathrm{NMR} 400 \mathrm{MHz}_{\mathrm{CDCl}}\right.$ ) $\delta: 13.93(\mathrm{~s}, 1 \mathrm{H}, \mathrm{NH}), 4.42\left(\mathrm{q}, \mathrm{J} 7.0 \mathrm{~Hz}, 2 \mathrm{H}, \mathrm{CH}_{2}\right), 2.85(\mathrm{t}$, J $\left.5.3 \mathrm{~Hz}, 2 \mathrm{H}, \mathrm{CH}_{2}\right), 2.70\left(\mathrm{t}, J 5.3 \mathrm{~Hz}, 2 \mathrm{H}, \mathrm{CH}_{2}\right), 1.87-1.79\left(\mathrm{~m}, 4 \mathrm{H}, 2 \times \mathrm{CH}_{2}\right), 1.43\left(\mathrm{t}, J 7.0 \mathrm{~Hz}, 3 \mathrm{H}, \mathrm{CH}_{3}\right) .{ }^{13} \mathrm{C} \mathrm{NMR}(100$ $\mathrm{MHz}_{2} \mathrm{CDCl}_{3}$ ) $\delta: 166.9 ; 155.1 ; 145.8 ; 132.2 ; 130.1 ; 117.0 ; 113.5 ; 61.7 ; 26.1 ; 24.5 ; 22.6 ; 22.4 ; 14.1 . U_{\max }$ (ATR) / $\mathrm{cm}^{-1}$ : 2938, 2229, 1655, 1551, 1318, 1251, 1109, 812. HRMS (ESI) $\mathrm{m} / \mathrm{z}[\mathrm{M}-\mathrm{H}]^{-}$: calcd for $\mathrm{C}_{13} \mathrm{H}_{14} \mathrm{~N}_{2} \mathrm{O}_{2} \mathrm{~S}_{2}$ : 293.0418 ; found : 293.0428.

1-Methyl-6,7-dihydro-1H-pyrazolo[3,4-d]imidazo[1,2-a]pyrimidin-4(8H)-one (13). ${ }^{29}$ White solid; Yield: $16 \%$; $\mathrm{mp}>250^{\circ} \mathrm{C}$ (Lit. $>250{ }^{\circ} \mathrm{C}$ ) ${ }^{1} \mathrm{H}$ NMR $\left(400 \mathrm{MHz}, \mathrm{DMSO}-d_{6}\right) \delta: 8.08(\mathrm{~s}, \mathrm{H}, \mathrm{NH}), 7.73(\mathrm{~s}, 1 \mathrm{H}, \mathrm{H}$ arom), $4.01(\mathrm{t}, J 8.5 \mathrm{~Hz}$, $\left.2 \mathrm{H}, \mathrm{CH}_{2}\right), 3.71-3.59\left(\mathrm{~m}, 5 \mathrm{H}, \mathrm{CH}_{2}, \mathrm{CH}_{3}\right) .{ }^{13} \mathrm{C} \mathrm{NMR}\left(100 \mathrm{MHz}, \mathrm{DMSO}-d_{6}\right) \delta: 157.7 ; 156.1 ; 154.7 ; 133.8 ; 99.8$; 41.6 ; 38.8 ; 33.4. $U_{\max }\left(\right.$ ATR) / $\mathrm{cm}^{-1}: 3211,2922,1704,1681,1375,1092,770$. HRMS (ESI) $\mathrm{m} / z$ [M-H] : calcd for $\mathrm{C}_{8} \mathrm{H}_{9} \mathrm{ON}_{5}: 190.0729 ;$ found : 190.0723 .

2,3-Dihydroimidazo[1,2-a]pyrido[2,3-d]pyrimidin-5(1H)-one (14). ${ }^{30}$ White solid; Yield: $57 \%$; $\mathrm{mp} 309{ }^{\circ} \mathrm{C}$ (Lit. $\left.{ }^{30} 309-312{ }^{\circ} \mathrm{C}\right),{ }^{1} \mathrm{H}$ NMR $\left(400 \mathrm{MHz}, \mathrm{DMSO}-d_{6}\right) \delta: 8.61$ (dd, J $2 \mathrm{~Hz}, J 4.4 \mathrm{~Hz}, 1 \mathrm{H}, \mathrm{H}$ arom), $8.23(\mathrm{~m}, 2 \mathrm{H}, \mathrm{H}$ arom, $\mathrm{NH}), 7.11$ (dd, J $4.4 \mathrm{~Hz}, J 8 \mathrm{~Hz}, 1 \mathrm{H}, \mathrm{H}$ arom), 4.12 (t, J $\left.8.4 \mathrm{~Hz}, 2 \mathrm{H}, \mathrm{CH}_{2}\right), 3.63$ (t, J $\left.8.4 \mathrm{~Hz}, 2 \mathrm{H}, \mathrm{CH}_{2}\right) .{ }^{13} \mathrm{C} \mathrm{NMR}(100$ $\mathrm{MHz}$, DMSO- $\left.d_{6}\right) \delta: 161.0 ; 160.3 ; 156.5 ; 155.0 ; 135.0 ; 117.5 ; 111.8 ; 42.1 . \mathrm{U}_{\max }(\mathrm{ATR}) / \mathrm{cm}^{-1}: 3464,3082$, $2922,1655,1537,1348,1153$. HRMS (ESI) $m / z[M+H]^{+}:$calcd for $\mathrm{C}_{9} \mathrm{H}_{8} \mathrm{ON}_{4}: 189.0776$; found : 189.0777.

General procedure for reaction of iminodithiazoles with 2-aminobenzylamine, synthesis of compounds $6,7,10$ and 15 . In a sealed vial, a solution of imino ester $(0.66 \mathrm{mmol})$ and commercially avalaible 2aminobenzylamine $(0.16 \mathrm{~g}, 1.32 \mathrm{mmol})$ in THF $(3 \mathrm{~mL})$ was heated under microwave irradiations at $120^{\circ} \mathrm{C}$ until all of the imine had been consumed (tlc control). After cooling, the solvent was evaporated off and the residue was purified by column chromatography to afford the various compounds $6,7,10$ and 15 .

1,2,3,4,6,11-Hexahydro-13-thia-5a,11,12-triaza-indeno[1,2-b]anthracen-5-one (6). Orange solid; Yield: 7\%; $\mathrm{mp} 347^{\circ} \mathrm{C} ;{ }^{1} \mathrm{H}$ NMR (400 MHz, DMSO-d $\left.)\right) \delta: 10.36(\mathrm{~s}, 1 \mathrm{H}, \mathrm{NH}), 7.27$ (d, J 7.4 Hz, 1H, Harom), 7.22 (t, J 7.6 Hz, $1 \mathrm{H}, \mathrm{H}$ arom), 7.01-6.97 (m, 2H, H arom), 5.05 (s, 2H CH $\left.\mathrm{CH}_{2}\right), 2.79\left(\mathrm{~s}, 2 \mathrm{H}, \mathrm{CH}_{2}\right), 2.62\left(\mathrm{~s}, 2 \mathrm{H}, \mathrm{CH}_{2}\right), 1.75(\mathrm{~s}, 4 \mathrm{H}$, $\left.2 \mathrm{xCH}_{2}\right) .{ }^{13} \mathrm{C}$ NMR (400 MHz, DMSO- $\left.d_{6}\right) \delta: 165.2 ; 146.9 ; 135.9 ; 135.2 ; 130.1 ; 128.2 ; 126.2 ; 126.1,121.9,116.2 ;$ $114.7 ; 113.5 ; 41.5 ; 24.8 ; 23.8 ; 22.2 ; 21.4 . u_{\max }\left(\right.$ ATR) $/ \mathrm{cm}^{-1}: 3464,3082,2922,1655,1537,1348,1153$. HRMS (ESI) $m / z[\mathrm{M}+\mathrm{H}]^{+}$: calcd for $\mathrm{C}_{17} \mathrm{H}_{15} \mathrm{ON}_{3} \mathrm{~S}: 310.1014$; found : 310.1017 .

2-[(Quinazoline-2-carbothioyl)-amino]-4,5,6,7-tetrahydro-benzo[b]thiophene-3-carboxylic acid ethyl ester (7). Orange oil; Yield: $12 \% ;{ }^{1} \mathrm{H}$ RMN $\left(400 \mathrm{MHz}, \mathrm{CDCl}_{3}\right) \delta: 15.35(\mathrm{~s}, 1 \mathrm{H}, \mathrm{NH}), 9.63(\mathrm{~s}, 1 \mathrm{H}), 8.34(\mathrm{~d}, J 8.5 \mathrm{~Hz}, 1 \mathrm{H}, \mathrm{H}$ arom), 8.07-8.01 (m, 2H, 2x H arom), $7.78\left(\mathrm{t}, J 7.8 \mathrm{~Hz}, \mathrm{H}, \mathrm{H}\right.$ arom) 4.51 (q, J 7.1 Hz, 2H, $\left.\mathrm{CH}_{2}\right), 2.90(\mathrm{t}, J 4.9 \mathrm{~Hz}$, $\left.2 \mathrm{H}, \mathrm{CH}_{2}\right), 2.73\left(\mathrm{t}, J 4.9 \mathrm{~Hz}, 2 \mathrm{H}, \mathrm{CH}_{2}\right), 1.87-1.81\left(\mathrm{~m}, 4 \mathrm{H}, 2 \mathrm{xCH}_{2}\right), 1.47\left(\mathrm{t}, J 7.1 \mathrm{~Hz}, 3 \mathrm{H}, \mathrm{CH}_{3}\right) .{ }^{13} \mathrm{C} \mathrm{NMR}(100 \mathrm{MHz}$, $\left.\mathrm{CDCl}_{3}\right) \delta: 183.08 ; 166.25 ; 160.99 ; 154.55 ; 149.82 ; 147.98 ; 135.09 ; 132.20 ; 129.72 ; 129.50 ; 127.77$; $127.31 ; 124.43 ; 116.67 ; 60.79 ; 26.36 ; 24.53 ; 22.80 ; 22.75 ; 14.34$. HRMS (ESI) $\mathrm{m} / z[\mathrm{M}+\mathrm{H}]^{+}:$calcd for $\mathrm{C}_{20} \mathrm{H}_{19} \mathrm{O}_{2} \mathrm{~N}_{3} \mathrm{~S}_{2}: 398.0997$; found : 398.0995.

2-[(Quinazoline-2-carbothioyl)-amino]-thiophene-3-carboxylic acid methyl ester (10). Orange Oil, Yield: 14\%; ${ }^{1} \mathrm{H} \mathrm{NMR}\left(400 \mathrm{MHz}, \mathrm{CDCl}_{3}\right) \delta: 15.61(\mathrm{~s}, 1 \mathrm{H}, \mathrm{NH}), 9.63(\mathrm{~s}, 1 \mathrm{H}, \mathrm{H}), 8.34(\mathrm{~d}, 1 \mathrm{H}, J 8.8 \mathrm{~Hz}, \mathrm{H}$ arom), 8.00-8.10 (m, 2H, $\mathrm{H}$ arom), 7.78 (t, 1H, J $7.7 \mathrm{~Hz}, \mathrm{H}$ arom), 7.42 (d, 1H, J $5.9 \mathrm{~Hz}, \mathrm{Harom}), 6.89$ (d, 1H, J $5.9 \mathrm{~Hz}, \mathrm{H}$ arom), 4.04 (s, 3H, $\left.\mathrm{OCH}_{3}\right) .{ }^{13} \mathrm{C} \mathrm{NMR}\left(100 \mathrm{MHz}, \mathrm{CDCl}_{3}\right) \delta: 184.2 ; 165.6 ; 161.0 ; 149.6 ; 135.1 ; 129.7 ; 129.6 ; 127.3 ; 124.6 ; 124.5$, $117.5 ; 115.9 ; 52.1 . \mathrm{U}_{\max }\left(\right.$ ATR) $/ \mathrm{cm}^{-1}: 3344,3107,2950,1680,1538,1245,723$. HRMS (ESI) $\mathrm{m} / \mathrm{z}[\mathrm{M}+\mathrm{H}]^{+}: \mathrm{calcd}$ for $\mathrm{C}_{15} \mathrm{H}_{11} \mathrm{~N}_{3} \mathrm{O}_{2} \mathrm{~S}_{2}: 329.02927$; found 329.0284 .

1-Methyl-5,10-dihydro-1H-1,2,4a,10,11-pentaaza-cyclopenta[b]anthracen-4-one (15). White solid; Yield: 24\%; mp >350 $\mathrm{C} ;{ }^{1} \mathrm{H}$ NMR (400 MHz, DMSO- $\left.d_{6}\right) \delta: 10.59(\mathrm{~s}, 1 \mathrm{H}, \mathrm{NH}), 7.87$ (s, 1H, H arom), $7.3(\mathrm{~d}, J 7.4 \mathrm{~Hz}, 1 \mathrm{H}$, 
$\mathrm{H}$ arom), 7.22 (t, J $7.6 \mathrm{~Hz}, 1 \mathrm{H}, \mathrm{H}$ arom), 7.01-6.97 (m, 2H, Harom), 5.07 (s, 2H CH 2$), 3.61\left(\mathrm{~s}, 3 \mathrm{H}, \mathrm{CH}_{3}\right) .{ }^{13} \mathrm{CNMR}^{\mathrm{N}}$ $\left(400 \mathrm{MHz}, \mathrm{DMSO}-d_{6}\right) \delta: 156.9 ; 152.1 ; 149.4 ; 135.4 ; 134.3 ; 128.4 ; 126.4 ; 122.4 ; 116.8 ; 113.9 ; 100.4 ; 41.9$ ; 33,4. $U_{\max }$ (ATR) / $\mathrm{cm}^{-1}: 3231 ; 2923 ; 1685 ; 1533 ; 749$. HRMS (ESI) $\mathrm{m} / z[\mathrm{M}+\mathrm{H}]^{+}$: calcd for $\mathrm{C}_{13} \mathrm{H}_{11} \mathrm{ON}_{5}$ : 254.1042 ; found : 254.1040 .

\section{Acknowledgements}

The authors are gratefull to the French cancer league (Comité 17 de la Ligue Nationale contre le Cancer) for financial support and to "Cancéropôle Grand Ouest, axe Valorisation des produits de la mer en cancérologie" for scientific support. They also thank Antoine Bonnet for NMR experiments (Centre Commun d'Analyses LIENSs, university of La Rochelle).

\section{References}

1. Li, H.; Chen, C.; Xu, S.; Cao, X. J. Chem. 2013, 2013, e692074.

2. Hu, Y.-G.; Zheng, A.-H.; Li, G.-J.; Dong, M.-Z.; Ye, F.; Sun, F.; Liu, Z.-Y.; Li, W. J. Heterocycl. Chem. 2014, 51, E84-E88.

https://doi.org/10.1002/ihet.1823

3. Bozorov, K.; Zhao, J.-Y.; Elmuradov, B.; Pataer, A.; Aisa, H. A. Eur. J. Med. Chem. 2015, 102, 552-573. https://doi.org/10.1016/j.ejmech.2015.08.018

4. Elrazaz, E. Z.; Serya, R. A. T.; Ismail, N. S. M.; Abou El Ella, D. A.; Abouzid, K. A. M. Future J. Pharm. Sci. 2015, 1, 33-41.

5. Ismail; Kuthati, B.; Thalari, G.; Bommarapu, V.; Mulakayala, C.; Chitta, S. K.; Mulakayala, N. Bioorg. Med. Chem. Lett. 2017, 27, 1446-1450.

https://doi.org/10.1016/i.bmcl.2017.01.088

6. Cohen, A.; Suzanne, P.; Lancelot, J.-C.; Verhaeghe, P.; Lesnard, A.; Basmaciyan, L.; Hutter, S.; Laget, M.; Dumètre, A.; Paloque, L.; Deharo, E.; Crozet, M. D.; Rathelot, P.; Dallemagne, P.; Lorthiois, A.; Sibley, C. H.; Vanelle, P.; Valentin, A.; Mazier, D.; Rault, S.; Azas, N. Eur. J. Med. Chem. 2015, 95, 16-28.

https://doi.org/10.1016/j.ejmech.2015.03.011

7. Pisal, M. M.; Nawale, L. U.; Patil, M. D.; Bhansali, S. G.; Gajbhiye, J. M.; Sarkar, D.; Chavan, S. P.; Borate, H. B. Eur. J. Med. Chem. 2017, 127, 459-469.

https://doi.org/10.1016/j.ejmech.2017.01.009

8. Endo, Y.; Kawai, K.; Asano, T.; Amano, S.; Asanuma, Y.; Sawada, K.; Onodera, Y.; Ueo, N.; Takahashi, N.; Sonoda, Y.; Kamei, N.; Irie, T. Bioorg. Med. Chem. Lett. 2015, 25, 1910-1914.

https://doi.org/10.1016/i.bmcl.2015.03.031

9. Endo, Y.; Kawai, K.; Asano, T.; Amano, S.; Asanuma, Y.; Sawada, K.; Ogura, K.; Nagata, N.; Ueo, N.; Takahashi, N.; Sonoda, Y.; Kamei, N. Bioorg. Med. Chem. Lett. 2015, 25, 649-653.

https://doi.org/10.1016/j.bmcl.2014.11.090

10. Kim, Y.; Kim, J.; Kim, S.; Ki, Y.; Seo, S. H.; Tae, J.; Ko, M. K.; Jang, H.-S.; Lim, E. J.; Song, C.; Cho, Y.; Koh, H.-Y.; Chong, Y.; Choo, I. H.; Keum, G.; Min, S.-J.; Choo, H. Eur. J. Med. Chem. 2014, 85, 629-637. https://doi.org/10.1016/j.ejmech.2014.08.027 
11. Di Fruscia, P.; Zacharioudakis, E.; Liu, C.; Moniot, S.; Laohasinnarong, S.; Khongkow, M.; Harrison, I. F.; Koltsida, K.; Reynolds, C. R.; Schmidtkunz, K.; Jung, M.; Chapman, K. L.; Steegborn, C.; Dexter, D. T.; Sternberg, M. J. E.; Lam, E. W.-F.; Fuchter, M. J. ChemMedChem 2015, 10, 69-82.

https://doi.org/10.1002/cmdc.201402431

12. Sundriyal, S.; Moniot, S.; Mahmud, Z.; Yao, S.; Di Fruscia, P.; Reynolds, C. R.; Dexter, D. T.; Sternberg, M. J. E.; Lam, E. W.-F.; Steegborn, C.; Fuchter, M. J. J. Med. Chem. 2017, 60, 1928-1945.

https://doi.org/10.1021/acs.jmedchem.6b01690

13. Wu, C.-H.; Coumar, M. S.; Chu, C.-Y.; Lin, W.-H.; Chen, Y.-R.; Chen, C.-T.; Shiao, H.-Y.; Rafi, S.; Wang, S.Y.; Hsu, H.; Chen, C.-H.; Chang, C.-Y.; Chang, T.-Y.; Lien, T.-W.; Fang, M.-Y.; Yeh, K.-C.; Chen, C.-P.; Yeh, T.-K.; Hsieh, S.-H.; Hsu, J. T.-A.; Liao, C.-C.; Chao, Y.-S.; Hsieh, H.-P. J. Med. Chem. 2010, 53, 7316-7326. https://doi.org/10.1021/jm100607r

14. Assy, M. G.; Sherif, M. H.; Amr, A. E.-G. E.; Abdelsalam, O. I.; Al-Omar, M. A.; Abdalla, M. M.; Ragab, I. J. Heterocycl. Chem. 2013, 50, 766-773.

https://doi.org/10.1002/jhet.1554

15. Dzhavakhishvili, S. G.; Gorobets, N. Y.; Shishkina, S. V.; Shishkin, O. V.; Desenko, S. M.; Groth, U. M.

J. Comb. Chem. 2009, 11, 508-514.

https://doi.org/10.1021/cc9000373

16. Hesse, S.; Perspicace, E.; Kirsch, G. Tetrahedron Lett. 2007, 48, 5261-5264.

https://doi.org/10.1016/i.tetlet.2007.05.136

17. El-Sherief, H. A. H.; El-Naggar, G. M.; Hozien, Z. A.; El-Sawaisi, S. M. J. Heterocycl. Chem. 2008, 45, 467473.

https://doi.org/10.1002/jhet.5570450226

18. Pokhodylo, N. T.; Matiychuk, V. S.; Obushak, M. D. Tetrahedron 2008, 64, 1430-1434. https://doi.org/10.1016/i.tet.2007.11.045

19. Appel, R.; Janssen, H.; Siray, M.; Knoch, F. Chem. Ber. 1985, 118, 1632-1643.

https://doi.org/10.1002/cber.19851180430

20. Konstantinova, L. S.; Bol'shakov, O. I.; Baranovsky, I. V.; Bogacheva, A. M.; Strunyasheva, V. V.; Rakitin, O. A. Mendeleev Commun. 2015, 25, 427-428.

https://doi.org/10.1016/i.mencom.2015.11.009

21. Koutentis, P. A.; Koyioni, M.; Michaelidou, S. S. Org. Biomol. Chem. 2012, 11, 621-629. https://doi.org/10.1039/C2OB26993G

22. Kalogirou, A. S.; Koutentis, P. A. Molecules 2015, 20, 14576-14594.

https://doi.org/10.3390/molecules200814576

23. Steinhardt, R. C.; Rathbun, C. M.; Krull, B. T.; Yu, J. M.; Yang, Y.; Nguyen, B. D.; Kwon, J.; McCutcheon, D. C.; Jones, K. A.; Furche, F.; Prescher, J. A. ChemBioChem 2017, 18, 96-100.

https://doi.org/10.1002/cbic.201600564

24. Konstantinova, L. S.; Bol'shakov, O. I.; Obruchnikova, N. V.; Laborie, H.; Tanga, A.; Sopéna, V.; Lanneluc, I.; Picot, L.; Sablé, S.; Thiéry, V.; Rakitin, O. A. Bioorg. Med. Chem. Lett. 2009, 19, 136-141.

https://doi.org/10.1016/j.bmcl.2008.11.010

25. Kim, K. Sulfur Rep. 1998, 21, 147-207.

https://doi.org/10.1080/01961779808047935

26. Chang, Y.-G.; Kim, K. Synlett 2002, 2002, 1423-1426.

27. Pereira, M. de F.; Thiéry, V.; Besson, T. J. Sulfur Chem. 2006, 27, 49-55.

https://doi.org/10.1080/17415990500493213 
28. De Fatima Pereira, M.; Thiéry, V.; Besson, T. Tetrahedron 2007, 63, 847-854. https://doi.org/10.1016/j.tet.2006.11.028

29. Wahab Khan, M.; Uddin, M. K.; Ali, M.; Rahman, M. S.; Rashid, M. A.; Chowdhury, R. J. Heterocycl. Chem. 2014, 51, E216-E221. https://doi.org/10.1002/ihet.1891

30. Urleb, U.; Stanovnik, B.; Tišler, M. J. Heterocycl. Chem. 1990, 27, 643-646.

https://doi.org/10.1002/ihet.5570270331 